\title{
13. Korrektur des Hypertelorismus
}

\author{
E. ScHMrd, W. WIDMATER und H. REICHERT \\ Abteilung für Gesichts-, Plastische und Wiederherstellungschirurgie \\ Marienhospital Stuttgart
}

In den letzten 15 Jahren haben wir uns mit den Problemen der Behandlung des Hypertelorismus auseinandergesetzt und eigene Techniken zur Korrektur desselben entwickelt.

Zum Teil wurden unsere Behandlungsverfahren durch die Ideen von Tessier günstig beeinflußt, der sich vor allem der Behandlung des Hypertelorismus seit einem Jahrzehnt besonders angenommen hat.

Mit der Ausstellung ist beabsichtigt, die Entwicklung unseres operationstechnischen Vorgehens bis zum gegenwärtigen Stand aufzuzeichnen und die erreichten Ergebnisse darzustellen.

Die Entwicklung der Operationstechniken für den Hypertelorismus hat darüber hinaus Impulse gesetzt für die Behandlung schwerer Orbitafrakturen und anderer Schädelmißbildungen.

Über ihre Behandlungsmöglichkeiten wird ebenfalls an Hand von Zeichnungen und Dokumentationsmaterial berichtet.

(S. auch Filmbericht Nr.9.) 